\title{
Dietary patterns and risk of heart disease in populations from different geographical locations in Saudi Arabia
}

\author{
N. Al Moraie, G. Lietz and C. J. Seal \\ Human Nutrition Research Centre, Food Quality and Health Group, School of Agriculture, Food \& Rural Development, \\ Newcastle University, Newcastle upon Tyne NE1 7RU, UK
}

The food situation in Saudi Arabia has markedly changed during the last two decades. A nutrition transition is taking place in the country in which traditional foods are being replaced by fast foods high in fat, sugar, and salt. Since one important modifiable risk factor of cardiovascular disease (CVD) is dietary intake. It is important to monitor these changes in order to estimate the potential effects of this dietary transition as CVD risk. Saudi Arabia has a wide geographical variation with its two major cities located in contrasting regions where local food availability is very different. Therefore, this study was designed to investigate dietary intake in two samples of adults living in different geographic locations (coastal and internal areas) of Saudi Arabia. 229 Saudi men and women aged 18-65 years were recruited from King Abdul-Aziz University, in the coastal city of Jeddah (50 men, 50 women) and Umm Al Qura University, in the inland city of Makkah (73 men, 56 women). All participants completed detailed three consecutive day food records and an assessment questionnaire that included question on lifestyle practices and socioeconomic status. Height and weight, waist and hip circumference were measured in order to calculate body mass index $\left(\mathrm{kg} / \mathrm{m}^{2}\right)$, and waist/hip ratio, respectively. The mean (SD) ages of women and men were 32.1 (8.01) and 32.3 (8.71), respectively. BMI was lower in men and women from the coastal region $25.1(2.76)$ than the inland region 26.3 (3.21), respectively $(P<0.05)$. Smoking was more prevalent in the inland area $(36 \%)$ than coastal area $(17 \%)$, $(P<0.001)$. Men were more physically active than women. Daily energy and nutrient intake averaged for men and women for the coastal and inland regions are shown in the Table:

\begin{tabular}{|c|c|c|c|}
\hline Nutrient & $\begin{array}{c}\text { Coastal City Mean (SD) } \\
n=100\end{array}$ & $\begin{array}{c}\text { Inland City Mean (SD) } \\
n=129\end{array}$ & $P$ \\
\hline Energy $(\mathrm{kcal} / \mathrm{d})$ & $2005.3(325.21)$ & $2272.9(227.51)$ & $<0.05$ \\
\hline Total Fat ( $\%$ energy) & $35.8(6.16)$ & $32.9(4.53)$ & N.S \\
\hline SFA ( $\%$ energy) & $12.4(3.41)$ & $14.4(2.82)$ & $<0.05$ \\
\hline MUFA ( $\%$ energy) & $12.4(2.98)$ & $8.8(8.4,9.2)^{*}$ & $<0.05$ \\
\hline PUFA ( $\%$ energy) & $6.8(2.29)$ & $3.2(3.01,3.3)^{*}$ & $<0.05$ \\
\hline Protein ( $\%$ energy) & $16.4(3.19)$ & $15.2(2.86)$ & N.S \\
\hline Carbohydrates (\% energy) & $50.5(6.78)$ & $55.3(5.08)$ & $<0.05$ \\
\hline Fibre $(\mathrm{g} / \mathrm{d})$ & $10.1(5.04)$ & $8.9(3.26)$ & $<0.05$ \\
\hline Sodium $(\mathrm{g} / \mathrm{d})$ & $2.9(0.98)$ & $4.4(0.12)$ & $<0.05$ \\
\hline Potassium (g/d) & $2.6(0.85)$ & $3.1(0.79)$ & $<0.05$ \\
\hline Selenium $(\mu \mathrm{g} / \mathrm{d})$ & $53.7(28.77)$ & $43.1(16.58)$ & $<0.05$ \\
\hline Zinc (mg/d) & $8.2(2.31)$ & $9.9(2.23)$ & $<0.05$ \\
\hline Vitamin $A(\mu \mathrm{g} / \mathrm{d})$ & $591.3(512.9,681.5)^{*}$ & $550.2(225.86)$ & N.S \\
\hline Vitamin C (mg/d) & $51.4(43.6,60.6)^{*}$ & $54.1(47.8,60.9)^{*}$ & N.S \\
\hline Vitamin E (mg/d) & $4.8(4.4,5.3)^{*}$ & $4.5(1.37)$ & $<0.05$ \\
\hline
\end{tabular}

SFA indicates saturated fatty acids; MUFA, monounsaturated fatty acids; PUFA, polyunsaturated fatty acids.

*Geometric means $(95 \% \mathrm{CI})$, variables compared by Mann-Whitney tests. NS: not significant.

Subjects from the coastal city had a significantly lower energy intake but higher intakes of MUFA and PUFA, fibre, selenium and vitamin $\mathrm{E}(P<0.05)$. In contrast they ate significantly less carbohydrates, sodium, potassium and zinc. The results show marked differences in diet composition between coastal and internal areas which may affect CVD risk. The reasons for these differences require further investigation.

NAM is in receipt of a scholarship from the Saudi government. 